\title{
Pan-cancer landscape of CD274 (PD-L1) copy number changes in 244584 patient samples and the correlation with PD-L1 protein expression
}

\author{
Richard S.P. Huang (D) , ${ }^{1}$ Karthikeyan Murugesan, ${ }^{1}$ Meagan Montesion, ${ }^{1}$ \\ Dean C. Pavlick, ${ }^{1}$ Douglas A. Mata, ${ }^{1}$ Matthew C. Hiemenz, ${ }^{1}$ Brennan Decker, ${ }^{1}$ \\ Garrett Frampton, ${ }^{1}$ Lee A. Albacker, ${ }^{1}$ Jeffrey S. Ross ${ }^{1,2}$
}

To cite: Huang RS.P,

Murugesan K, Montesion M, et al. Pan-cancer landscape of CD274 (PD-L1) copy number changes in 244584 patient samples and the correlation with PD-L1 protein expression. Journal for ImmunoTherapy of Cancer 2021;9:e002680. doi:10.1136/jitc-2021-002680

- Additional supplemental material is published online only. To view, please visit the journal online (http://dx.doi.org/10. 1136/jitc-2021-002680).

Accepted 09 April 2021

Check for updates

(C) Author(s) (or their employer(s)) 2021. Re-use permitted under CC BY-NC. No commercial re-use. See rights and permissions. Published by BMJ.

${ }^{1}$ Foundation Medicine Inc, Cambridge, Massachusetts, USA ${ }^{2}$ Department of Pathology, State University of New York (SUNY) Upstate Medical University, Syracuse, New York, USA

Correspondence to Dr Richard S.P. Huang; rhuang@foundationmedicine. com

\section{ABSTRACT}

Introduction Several studies have shown clinical outcomes data that support the use of CD274 (PD-L1) copy-number (CN) gains and/or losses as a biomarker for immune checkpoint inhibitor (ICPI). Here, we present the landscape of CD274 CN changes across a large cohort of solid tumor cases and correlate these with PD-L1 protein expression by immunohistochemistry.

Methods We analyzed all cases that underwent comprehensive genomic profiling (CGP) testing at Foundation Medicine between August 2014 and June 2020. CD274 CN changes were correlated with PD-L1 expression in tumor types where there were Food and Drug Administration approved companion diagnostic (CDx) claims and the CDx assay was used to assess PD-L1 expression.

Results In all, 244584 samples representing 290 solid tumor types were included in the study. Overall, 17.6\% (42 983/244 584) had CD274 CN gains (>specimen ploidy), $44.6 \%$ (108 970/244 584) were CD274 CN neutral, and $37.9 \%$ (92 631/244 584) had CD274 CN loss. Using different $C N$ cut offs to define $C D 274$ positivity resulted in different prevalence estimates: ploidy $+1,17.4 \%$ (42 636/244 584); ploidy +2, 6.2\% (15 183/244 584); ploidy $+3,2.2 \%$ (5375/244 584); ploidy +4, 1.1\% (2712/244 584); and ploidy $+8,0.2 \%$ (434/244 584). The prevalence of $\mathrm{CN}$ changes and $\mathrm{CN}$ positivity varied based on tumor type. CD274 CN gains were significantly associated with PD-L1 positivity in NSCLC, urothelial carcinoma, breast carcinoma, cervical carcinoma, esophagus squamous cell carcinoma (SCC) and head and neck SCC (ORs 3.3, 3.0, 2.0, 4.5. 3.8, $8.4,1.4$, respectively; $p<0.05$ ) and with microsatellite instability status in only clinically relevant tumor types (gastric adenocarcinoma, colorectal adenocarcinoma, uterine endometrial adenocarcinoma, esophageal adenocarcinoma and gastroesophageal junction adenocarcinoma (OR: 5.2, $1.9,3.2,3.7$ and 6.5 , respectively; $p<0.05))$. Conversely, CD274 CN changes were not significantly correlated with tumor mutational burden in almost all the tumor types. Conclusion CD274 CN changes and PD-L1 expression were highly correlated in multiple tumor types. These prevalence data on $C D 274 \mathrm{CN}$ changes across a large cohort of different solid tumors can be used to design future clinical studies to assess whether $C D 274 \mathrm{CN}$ changes could be a potential biomarker for ICPI.

\section{INTRODUCTION}

Immune checkpoint inhibitors (ICPI) have revolutionized treatment options for cancer patients, and three biomarkers are currently approved by the United States Food and Drug Administration (FDA) as companion diagnostics (CDx) for ICPI. ${ }^{1}$ These include microsatellite instability (MSI) testing, where MSI-High (MSI-H) patients with solid tumors are eligible for pembrolizumab; tumor mutational burden (TMB) testing by comprehensive genomic profiling (CGP), where solid tumor patients with TMB $\geq 10$ mutations/ megabase (mut/Mb) (TMB-High, TMB-H) are also eligible for pembrolizumab; and PD-L1 expression measured by immunohistochemistry (IHC), where PD-L1 positive tumor cells or immunocytes in certain tumor types enable the selection of ICPI such as pembrolizumab, atezolizumab or nivolumab. ${ }^{2-6}$ One promising but not as well studied biomarker for ICPI is CD274 (PD-L1) gene copy number (CN) changes.

In clinically advanced Hodgkin lymphoma, a tumor type that generally responds well to ICPI, CD274 CN gains is almost always present. ${ }^{7-9}$ In addition, in a study using CGP, CD274 amplification (defined as ploidy +4 (CN 6)) was identified in $0.7 \%$ of patients across a large cohort of diverse solid tumors, and some evidence emerged indicating that CD274 amplification is a predictor of ICPI response. ${ }^{10}$ More recently, in the SAFIR02IMMUNO Randomized Phase II Trial, exploratory analysis has shown that $\mathrm{CD} 274$ amplification (defined as $\mathrm{CN} \geq 5$ ) and $\mathrm{CN}$ gains (CN 3-4) were predictors of durvalumab response in metastatic breast cancer. ${ }^{11}$ In nonsmall-cell lung cancer (NSCLC), Lamberti et $a l^{2}$ discovered that $C D 274$ loss was associated with a lower response rate and progression 
free survival when compared with patients without $C D 274$ loss. In another study, the authors concluded that $C D 274$ amplification (defined as $\mathrm{CD} 274$ to CEP9 ratio of at least 2.0 as determined by fluorescence in situ hybridization) was associated with response to nivolumab monotherapy in NSCLC patients. ${ }^{13}$ While these studies used different methodologies to detect $\mathrm{CD} 274 \mathrm{CN}$ changes, overall the data showed that increases in $\mathrm{CD} 274 \mathrm{CN}$ were associated with better response to ICPI and decreases in $\mathrm{CD} 274 \mathrm{CN}$ were associated with an attenuated response to ICPI.

Currently, evidence exists in the literature supporting CD274 CN gains and losses as a promising biomarker for ICPI; however, the landscape of $\mathrm{CD} 274 \mathrm{CN}$ changes in different tumor types has not been well described. In addition, while a strong correlation was observed in CD274 amplification (ploidy +4 (CN 6) with CGP) and PD-L1 positivity across solid tumor types, a more nuanced study of $\mathrm{CD} 274 \mathrm{CN}$ and PD-L1 expression is lacking. ${ }^{32}$ Here, we investigated the landscape of $\mathrm{CD} 274 \mathrm{CN}$ changes as detected by CGP in a large cohort of diverse solid tumor cases and correlated the $C D 274 \mathrm{CN}$ to the well-established ICPI biomarkers of PD-L1 IHC, MSI and TMB. We show that the prevalence of $\mathrm{CD} 274 \mathrm{CN}$ changes varied based on tumor type, and that $\mathrm{CD} 274 \mathrm{CN}$ changes and PD-L1 expression were highly correlated in multiple tumor types.

\section{MATERIALS AND METHODS Data collection}

We analyzed all cases that underwent CGP testing at Foundation Medicine between August 2014 and June 2020. Available clinical information for the patient samples were extracted from accompanying test requisition form and pathology reports.

All specimens were assigned a diagnosis by a boardcertified pathologist based on microscopic examination of a H\&E-stained slide from the formalin-fixed-paraffinembedded (FFPE) tissue, pathology report, and clinical information provided by the ordering physician.

\section{Comprehensive genomic profiling}

CGP was performed on hybridization-captured, adaptor ligation-based libraries using DNA extracted from FFPE tumor tissue in a Clinical Laboratory Improvement Amendments (CLIA)-certified and College of American Pathologists (CAP)-accredited laboratory (Foundation Medicine, Cambridge, Massachusetts, USA). The samples were sequenced for up to 324 cancer related genes and/ or select gene rearrangements. ${ }^{14}$

CN alterations were detected using a comparative genomic hybridization-like method applied to next generation sequencing data. ${ }^{14}{ }^{15}$ In the laboratory, each specimen was analyzed alongside a process-matched normal control (an internally validated mixture of 10 heterozygous diploid samples from the HapMap project), with custom algorithms to normalize the sequence coverage distribution across captured DNA regions.
Log-ratios of normalized coverage data for exonic, intronic, and SNP targets accounting for stromal admixture, as well as genome-wide SNP frequencies, were used to generate the profiles. Using circular binary segmentation, custom algorithms further clustered groups of targets and SNP frequencies to define upper and lower bounds of genomic segments. Empirical Bayesian algorithms employed a distribution of parameters including purity and base ploidy and probability matrices were derived using different statistical sampling methodologies to fit these data. Specimen level ploidy was estimated as described by Sun et $a l^{15}$. Computational models were reviewed by expert analysts for each sample. ${ }^{14} \mathrm{CD} 274$ $\mathrm{CN}$ gain was defined as any $\mathrm{CN}>$ ploidy of the specimen, CD274 CN neutral was any $\mathrm{CN}$ that equaled the ploidy of the specimen, and $\mathrm{CD} 274 \mathrm{CN}$ loss was any $\mathrm{CN}<$ ploidy of the specimen. We also explored prevalence rates of different $\mathrm{CN}$ cut offs (ploidy +1 (CN 3), ploidy $+2(\mathrm{CN} 4)$, ploidy +3 (CN 5), ploidy +4 (CN 6), and ploidy +8 (CN 10)) to define $\mathrm{CD} 274 \mathrm{CN}$ positivity.

TMB was determined on $0.8-1.1 \mathrm{Mb}$ of sequenced DNA and calculated as the number of non-driver somatic coding mut/Mb of genome sequenced. ${ }^{16}$ For TMB, we considered a TMB cut-off of at least $10 \mathrm{mut} / \mathrm{Mb}$ as TMB-H in our analysis based on the FDA pan-solid tumor CDx approval for pembrolizumab. ${ }^{17}$ MSI status was determined by analyzing 114 intronic homopolymer repeat loci for length variability, as previously described. ${ }^{18}$ MSI positivity was defined as MSI-H as per the pan-tumor approval for pembrolizumab. ${ }^{19}$

As an exploratory analysis, we hypothesized that the high rates of $\mathrm{CD} 274 \mathrm{CN}$ gains in cervix squamous cell carcinoma (SCG) and Head and Neck (HN) SCC could be due to human papillomavirus (HPV) infection, and therefore explored the HPV status of these patients. To determine the HPV status of the samples, we performed de novo assembly of non-human sequencing reads and BLASTn comparison against all viral nucleotide sequences in the NCBI RefSeq database were used to detect the presence of HPV genome sequences (Research Use Only).

\section{PD-L1 IHC}

PD-L1 IHC testing was run and interpreted by boardcertified pathologists according to the manufacturer instructions in a CLIA-certified and CAP-accredited laboratory (Foundation Medicine, Morrisville, North Carolina) for a subset of specimens in this cohort. ${ }^{2021}$ Specially, we examined the tumor types with a PD-L1 CDx approval: DAKO 22C3 PD-L1 assay for NSCLC (tumor proportion score cut-off $\geq 1$ ), cervical carcinoma (combined positive score (CPS) cut-off $\geq 1$ ), head and neck SCC (CPS cutoff $\geq 1$ ), gastric/gastroesophageal adenocarcinoma (CPS cut-off $\geq 1$ ), urothelial carcinoma (CPS cut-off $\geq 10$ ), and esophageal SCC (CPS cut-off $\geq 10$ ); and VENTANA SP142 PD-L1 assay for breast carcinoma at tumor infiltrating immune cell cut-off of $1 \%$. $^{22-24}$ 


\section{Analyses}

Statistical analyses were performed using R V.3.6.0 (R Foundation for Statistical Computing, Vienna, Austria) and Python V.2.7.16. ${ }^{25}{ }^{26}$ Differences among categorical variables were assessed using the Fisher's exact test. Statistical tests were two sided and multiple hypothesis testing correction was performed using the Benjamini-Hochberg procedure.

Currently, PD-L1 IHC is the gold standard for the tumor types with a PD-L1 CDx claim. We further sought to investigate the sensitivity, specificity, positive predictive value and negative predictive value of $\mathrm{CD} 274 \mathrm{CN}$ positivity (based on different $\mathrm{CN}$ cut-offs) when compared with PD-L1 IHC positivity.

\section{RESULTS}

\section{CD274 CN changes in various tumor types}

We analyzed $C D 274 \mathrm{CN}$ in 244584 solid tumor samples representing 290 solid tumor types. Overall, $17.6 \%$ $(42,983 / 244,584)$ had CD274 $\mathrm{CN}$ gains (CN >ploidy), $44.6 \%(108,970 / 244,584)$ were CD274 CN neutral (CN=ploidy), and 37.9\% (92,631/244,584) had CD274 CN loss (CN <ploidy) (online supplemental table 1).

Among tumor types with $\geq 1000$ samples, cervical SCC $(31.3 \%)$, lung small-cell undifferentiated carcinoma (27.0\%), and head and neck SCC (HNSCC) (25.7\%) had the highest frequencies of $C D 274 \mathrm{CN}$ gain (figure 1). Conversely, skin melanoma (8.1\%), pancreatic ductal adenocarcinoma (8.5\%), and glioblastoma (9.3\%) had the lowest prevalence of $\mathrm{CD} 274$ gain. In addition, in the cohort with $\mathrm{CD} 274 \mathrm{CN}$ gains, we observed the highest magnitude of $\mathrm{CN}$ gains in tumor types with SCC morphology (figure 2). A positive correlation of $\mathrm{CD} 274 \mathrm{CN}$ gains with $\mathrm{HPV}$ infection in cervical SCC and HNSCC was observed $(\mathrm{OR}=1.4$, $\mathrm{p}=0.17$; OR 3.8, $\left.\mathrm{p}=8.8 \times 10^{-31}\right)$. Pancreatic ductal adenocarcinoma $(56.3 \%)$, gallbladder adenocarcinoma $(55.5 \%)$, and cutaneous melanoma $(55.4 \%)$ had the highest frequencies of $\mathrm{CD} 274 \mathrm{CN}$ loss. This contrasted with cervical SCC $(14.4 \%)$, appendiceal adenocarcinoma $(16.4 \%)$, and uterine endometrial adenocarcinoma (16.6\%), which had the lowest frequencies of $\mathrm{CD} 274 \mathrm{CN}$ loss (figure 1).

In addition, we explored the prevalence of $C D 274 \mathrm{CN}$ positivity based on different $\mathrm{CN}$ cut offs. When using ploidy +1 (CN 3) as the cut-off, the overall positivity was $17.4 \%$ $(42,636 / 244,584)$; when using ploidy +2 (CN 4) as the cut-off, the overall positivity was $6.2 \%(15,183 / 244,584)$; when using ploidy $+3(\mathrm{CN} 5)$ as the cut-off, the overall positivity was $2.2 \%$ (5375/244 584); when using ploidy $+4(\mathrm{CN} 6)$ as the cut-off, the overall positivity was $1.1 \%$ (2712/244 584); and when using ploidy +8 (CN 10) as the cut-off, the overall positivity was $0.2 \%$ (434/244 584). CD274 CN positivity in different tumor types also varied using these different cut-offs (figure 3 and online supplemental table 2).

\section{CD274 CN correlation with PD-L1 IHC}

CD274 CN gains were highly correlated with PD-L1 IHC positive status in almost all tumor types where a CDx assay was available (figure 4A). Interestingly, HNSCC had the highest OR in this comparison and gastric/ esophageal adenocarcinoma which had the lowest OR $\left(8.44, \mathrm{p}=3.1 \times 10^{-2} ; 1.41, \mathrm{p}=8.7 \times 10^{-2} ;\right.$ respectively $)$. NSCLC, urothelial carcinoma, breast carcinoma, cervical carcinoma, and esophageal SCC all had a positive and significant OR $\left(3.29, \mathrm{p}=3.2 \times 10^{-173} ; 2.97, \mathrm{p}=3.2 \times 10^{-15} ; 1.96\right.$, $\mathrm{p}=1.6 \times 10^{-13} ; 4.51, \mathrm{p}=2.1 \times 10^{-5} ;$ and $3.81, \mathrm{p}=8.7 \times 10^{-2}$, respectively).

While CD274 CN changes were highly correlated with PD-L1 IHC status across multiple tumor types, at a population level, there was still a subset of patients in which they were not. Specifically, 4.6\% (1378/29887) of the overall cohort with CD274 CN and PD-L1 IHC data had CD274 gain but were PD-L1 negative (table 1). Conversely, 23.3\% (6953/29 887) had CD274 loss but were PD-L1 IHC positive (table 1).

When compared with PD-L1 IHC, CD274 CN positivity (at different $\mathrm{CN}$ cut-offs) is highly specific and has high positive predictive value (figure $4 \mathrm{~B}$ and online supplemental table 3). On the other hand, sensitivity and the negative predictive value is lower. Importantly, the sensitivity, specificity, positive predictive value, and negative predictive value varied depending on which cut-off we used to define $C D 274 \mathrm{CN}$ positivity and varied depending on tumor type/PD-L1 CDx assay and scoring algorithm used (figure 4B and online supplemental table 3).

\section{CD274 CN correlation with TMB and MSI}

CD274 CN gains were not significantly correlated with TMB-H in almost all $(98.6 \%, 286 / 290)$ tumor types. CD274 CN gain were significantly correlated with TMB in only four tumor types: lung adenocarcinoma, gastric adenocarcinoma, uterine endometrial adenocarcinoma, and bladder urothelial carcinoma (OR: 1.2, $\mathrm{p}=9.3 \times 10^{-6}$; 2.3, $\mathrm{p}=1.6 \times 10^{-5} ; 2.3, \mathrm{p}=4.7 \times 10^{-5}$; and $1.4, \mathrm{p}=4.6 \times 10^{-2}$, respectively) (figure $5 \mathrm{~A}$ ).

In the tumor types in which MSI is most clinically relevant (gastric adenocarcinoma, colorectal adenocarcinoma, uterine endometrial adenocarcinoma, esophageal adenocarcinoma, and gastroesophageal junction adenocarcinoma), we observed a significant correlation between $\mathrm{CD} 274 \mathrm{CN}$ gains and MSI-H status (OR: 5.2, $\mathrm{p}=4.9 \times 10^{-10} ; 1.9, \mathrm{p}=6.7 \times 10^{-8} ; 3.2, \mathrm{p}=2.1 \times 10^{-6} ; 3.7$, $\mathrm{p}=5.5 \times 10^{-3}$; and $6.5, \mathrm{p}=2.3 \times 10^{-2}$, respectively) (figure $5 \mathrm{~B}$ ). Most of the remaining tumor types did not have significant correlation between $\mathrm{CD} 274 \mathrm{CN}$ gains and MSI-H.

\section{DISCUSSION}

In this study, we present prevalence data on $C D 274 \mathrm{CN}$ losses, gains, and positivity (defined by different $C D 274$ CN cut-offs) in over 240000 patient samples across 290 solid tumor types. While Goodman et $a l^{10}$ previously presented data on CD274 amplification status (defined as ploidy +4 ) on a large cohort of patients, recent clinical data suggests that $\mathrm{CN}$ loss, CN gain, and amplification based on different cut offs can represent both negative 


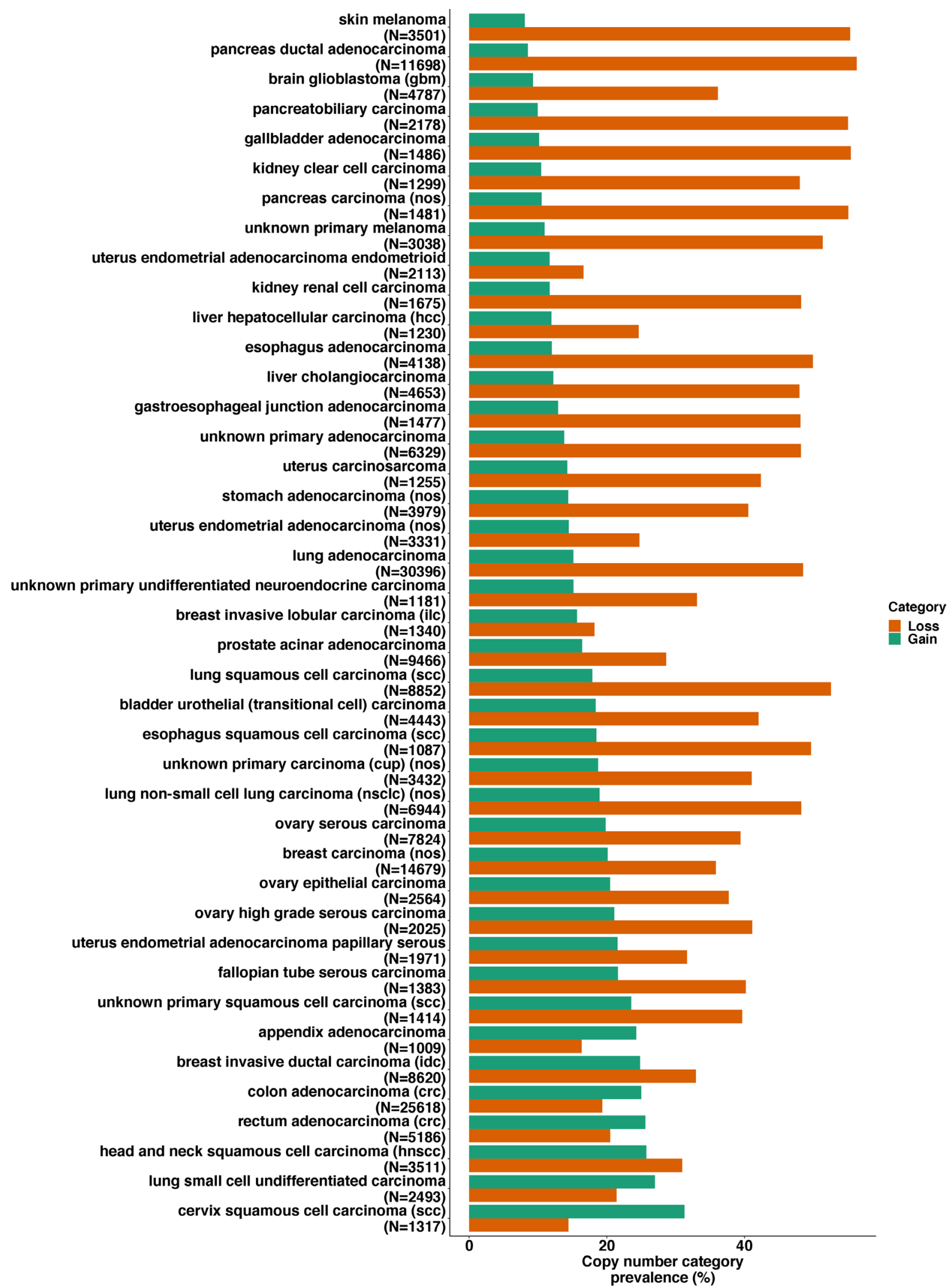

Figure 1 Prevalence of CD274 copy number (CN) gains and losses in different tumor types. Cervical SCC (31.3\%), lung small-cell undifferentiated carcinoma (27.0\%), and head and neck SCC (25.7\%) had the highest frequencies of CD274 CN gain; cutaneous melanoma (8.1\%), pancreatic ductal adenocarcinoma (8.5\%) and glioblastoma (9.3\%) had the lowest frequencies of CD274 gain. Pancreatic ductal adenocarcinoma (56.3\%), gallbladder adenocarcinoma (55.5\%) and cutaneous melanoma (55.4\%) had the highest frequencies of CD274 loss; cervical SCC (14.4\%), appendiceal adenocarcinoma (16.4\%) and uterine endometrial adenocarcinoma (16.6\%) had the lowest frequencies of CD274 loss. Only tumor types with at least 1000 samples are shown. 
pancreas ductal adenocarcinoma

brain glioblastoma $(\mathrm{gbm})$ pancreatobiliary carcinoma gallbladder adenocarcinoma kidney clear cell carcinoma pancreas carcinoma (nos) unknown primary melanoma uterus endometrial adenocarcinoma endometrioid kidney renal cell carcinoma liver hepatocellular carcinoma (hcc) esophagus adenocarcinoma

liver cholangiocarcinoma gastroesophageal junction adenocarcinoma unknown primary adenocarcinoma uterus carcinosarcoma stomach adenocarcinoma (nos) uterus endometrial adenocarcinoma (nos) lung adenocarcinoma unknown primary undifferentiated neuroendocrine carcinoma breast invasive lobular carcinoma (ilc) prostate acinar adenocarcinoma lung squamous cell carcinoma (scc) bladder urothelial (transitional cell) carcinoma esophagus squamous cell carcinoma (scc) unknown primary carcinoma (cup) (nos) lung non-small cell lung carcinoma (nsclc) (nos) ovary serous carcinoma breast carcinoma (nos) ovary epithelial carcinoma

ovary high grade serous carcinoma uterus endometrial adenocarcinoma papillary serous fallopian tube serous carcinoma unknown primary squamous cell carcinoma (scc) appendix adenocarcinoma breast invasive ductal carcinoma (idc) colon adenocarcinoma (crc) rectum adenocarcinoma (crc) head and neck squamous cell carcinoma (hnscc) lung small cell undifferentiated carcinoma cervix squamous cell carcinoma (scc)

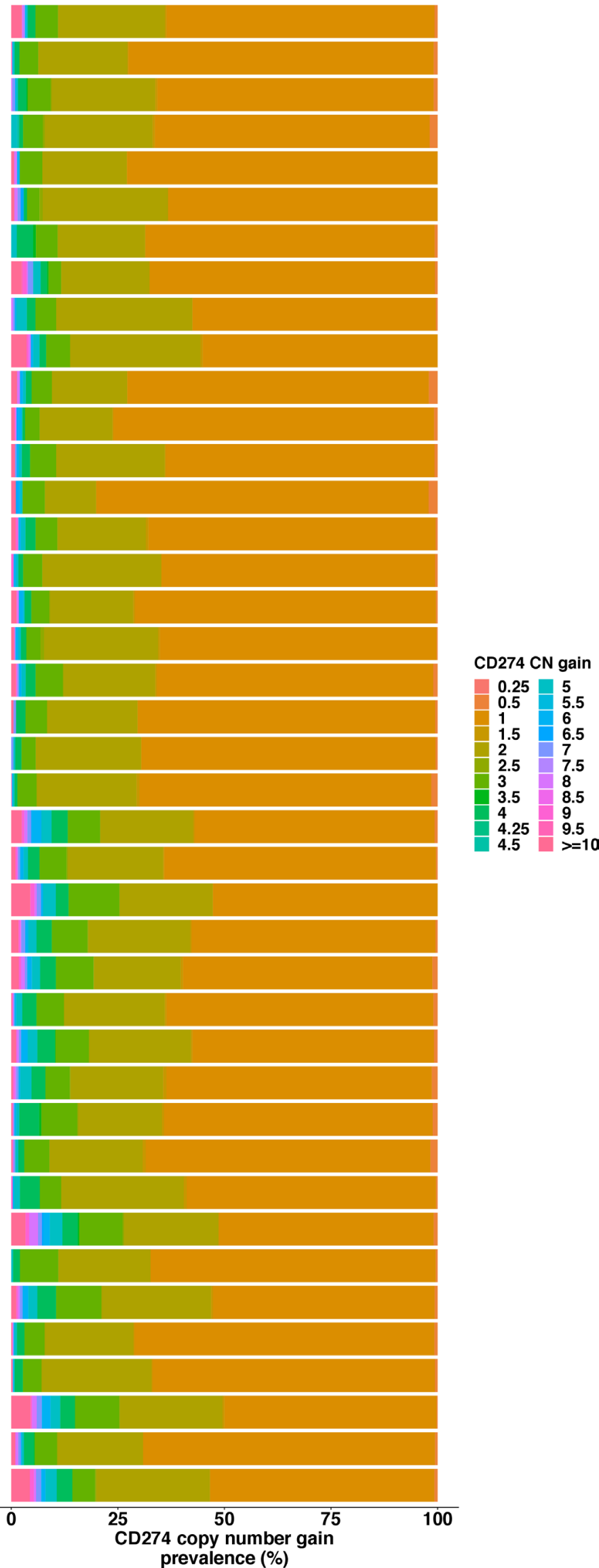

prevalence (\%)

Figure 2 Histogram of degree of CD274 CN gains in different tumor types. The highest levels of $\mathrm{CN}$ gains were observed in tumor types with SCC morphology. Only tumor types with at least 1000 samples are shown. CN, copy number. 
pancreas ductal adenocarcinoma gastroesophageal junction adenocarcinoma gallbladder adenocarcinoma skin melanoma esophagus adenocarcinoma brain glioblastoma (gbm) liver hepatocellular carcinoma (hcc) pancreas carcinoma (nos) pancreatobiliary carcinoma unknown primary melanoma

kidney clear cell carcinoma stomach adenocarcinoma (nos) liver cholangiocarcinoma unknown primary adenocarcinoma unknown primary undifferentiated neuroendocrine carcinoma breast invasive lobular carcinoma (ilc) prostate acinar adenocarcinoma uterus carcinosarcoma uterus endometrial adenocarcinoma endometrioid uterus endometrial adenocarcinoma (nos) lung adenocarcinoma kidney renal cell carcinoma

bladder urothelial (transitional cell) carcinoma uterus endometrial adenocarcinoma papillary serous ovary serous carcinoma colon adenocarcinoma (crc) ovary epithelial carcinoma ovary high grade serous carcinoma lung non-small cell lung carcinoma (nsclc) (nos)

lung squamous cell carcinoma (scc) appendix adenocarcinoma unknown primary carcinoma (cup) (nos) lung small cell undifferentiated carcinoma rectum adenocarcinoma (crc) breast carcinoma (nos) esophagus squamous cell carcinoma (scc) fallopian tube serous carcinoma unknown primary squamous cell carcinoma (scc) breast invasive ductal carcinoma (idc) head and neck squamous cell carcinoma (hnscc) cervix squamous cell carcinoma (scc)

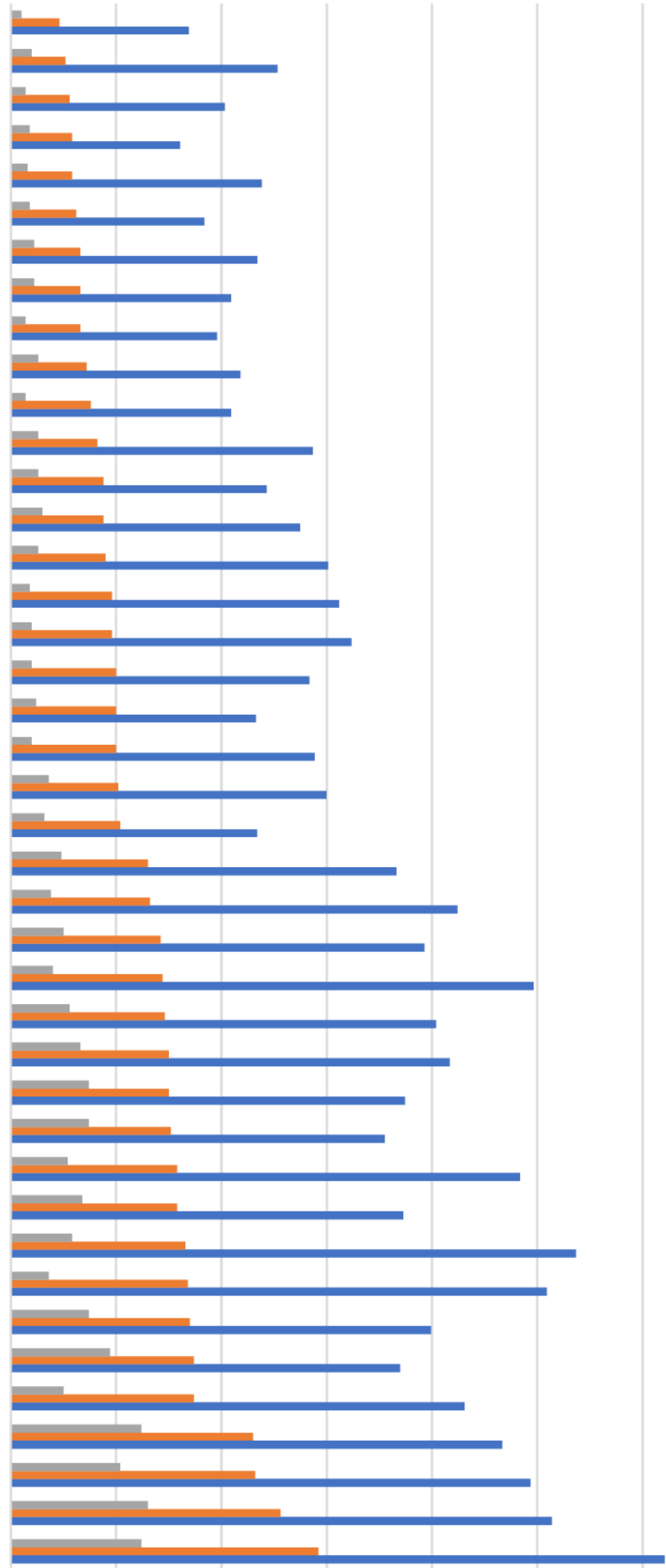

Figure 3 Histogram showing the prevalence of CD274 copy number $(C N)$ positivity at different CN cut-offs in different tumor types. Specially, CN cut-off at ploidy +1 (CN 3), ploidy $+2(\mathrm{CN} 4)$, and ploidy +3 (CN 5) are shown here. Only tumor types with at least 1000 samples are shown. 
A

Disease

NSCLC

Urothelial Carcinoma

Breast Carcinoma

Cervical Carcinoma

Esophageal SCC

HNSCC

Gastric/Esophageal

Adenocarcinoma

\section{B}

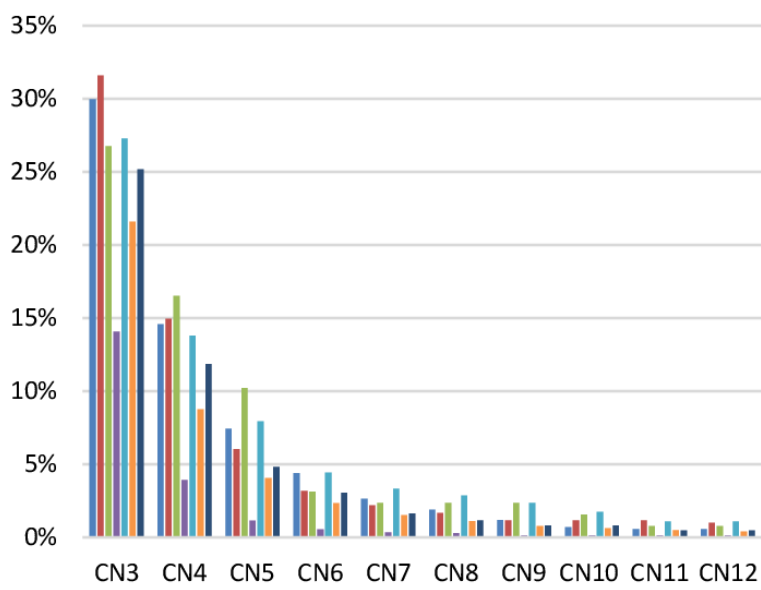

Positive Predictive Value

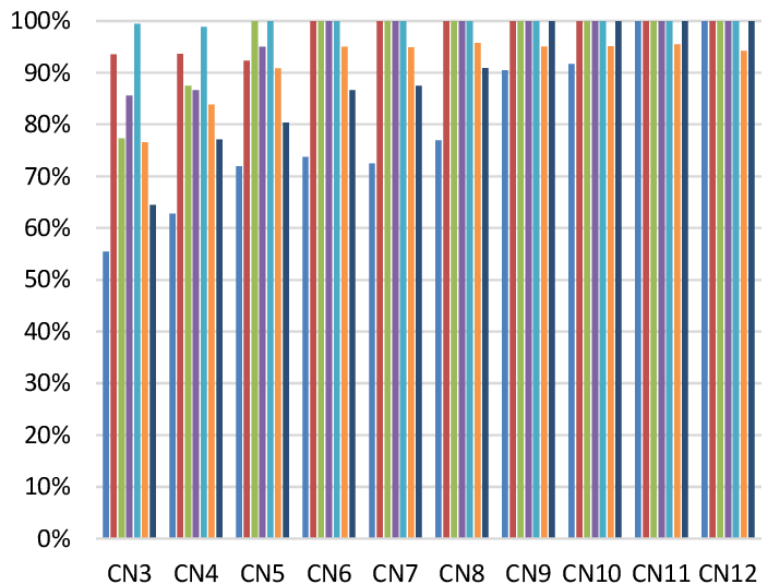

$$
\begin{aligned}
& \text { Breast carcinoma }(n=3833) \\
& \text { Esophageal SCC }(n=208) \\
& \text { HNSCC }(n=655) \\
& \text { Urothelial carcinoma }(n=1742)
\end{aligned}
$$

3.81

\begin{tabular}{ll|l}
3.29 & $3.2 \times 10^{-173}$ & \\
2.97 & $3.2 \times 10^{-15}$ & | \\
1.96 & $1.6 \times 10^{-13}$
\end{tabular}

$4.512 .1 \times 10^{-5}$

$1.2 \times 10^{-3}$

$3.1 \times 10^{-2}$

$8.7 \times 10^{-2}$

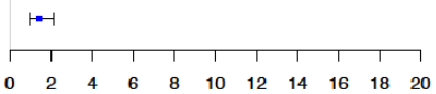

Specificity

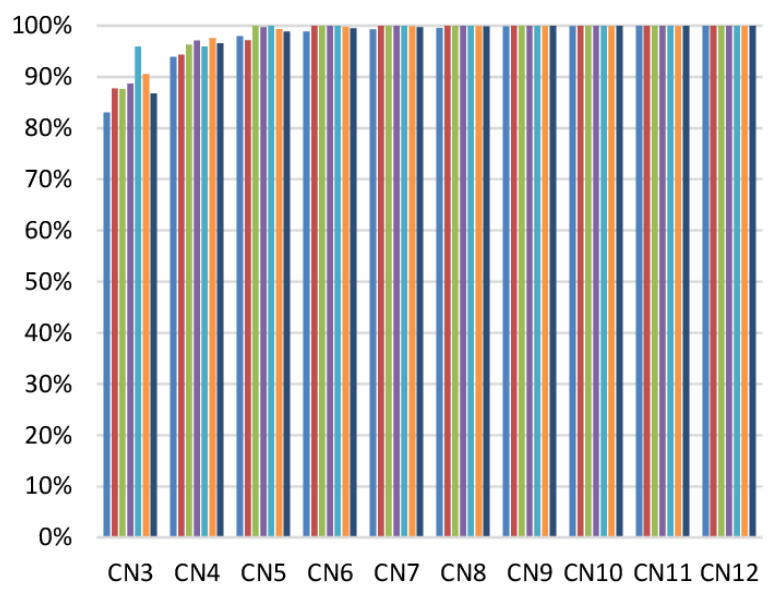

Negative Predictive Value

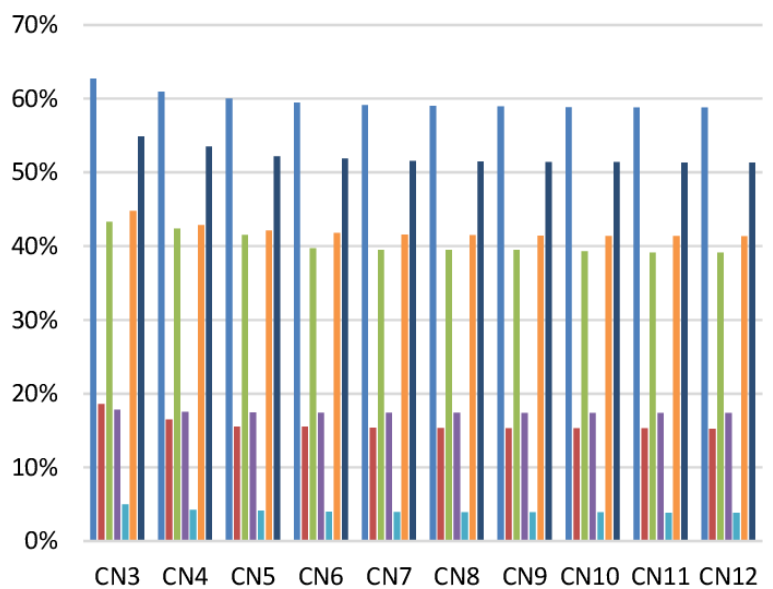

- Cervical carcinoma ( $\mathrm{n}=701)$

- Gastric/esophageal adenocarcinoma ( $n=1993)$

NSCLC ( $\mathrm{n}=20755)$

Figure 4 (A) OR forest plot of CD274 copy number (CN) gains and PD-L1 immunohistochemistry (IHC) positivity. CD274 CN gains were highly correlated with PD-L1 IHC positivity in almost all tumor types where a companion diagnostic assay was available. (B) Sensitivity, specificity, positive predictive value, and negative predictive value of CD274 CN positivity (defined at different cut-offs) when compared with PD-L1 IHC positivity. When compared with PD-L1 IHC, CD274 CN positivity is highly specific and has high positive predictive value. On the other hand, sensitivity and the negative predictive value is lower. Importantly, the sensitivity, specificity, positive predictive value, and negative predictive value varied depending on which cut-off we used to define $C D 274 \mathrm{CN}$ positivity and varied depending on tumor type/PD-L1 CDx assay and scoring algorithm used. CN $3=$ ploidy +1 , CN 4=ploidy +2 , CN 5=ploidy +3 , and so forth. HNSCC, head and neck SCC; NSCLC, non-small-cell lung cancer. 

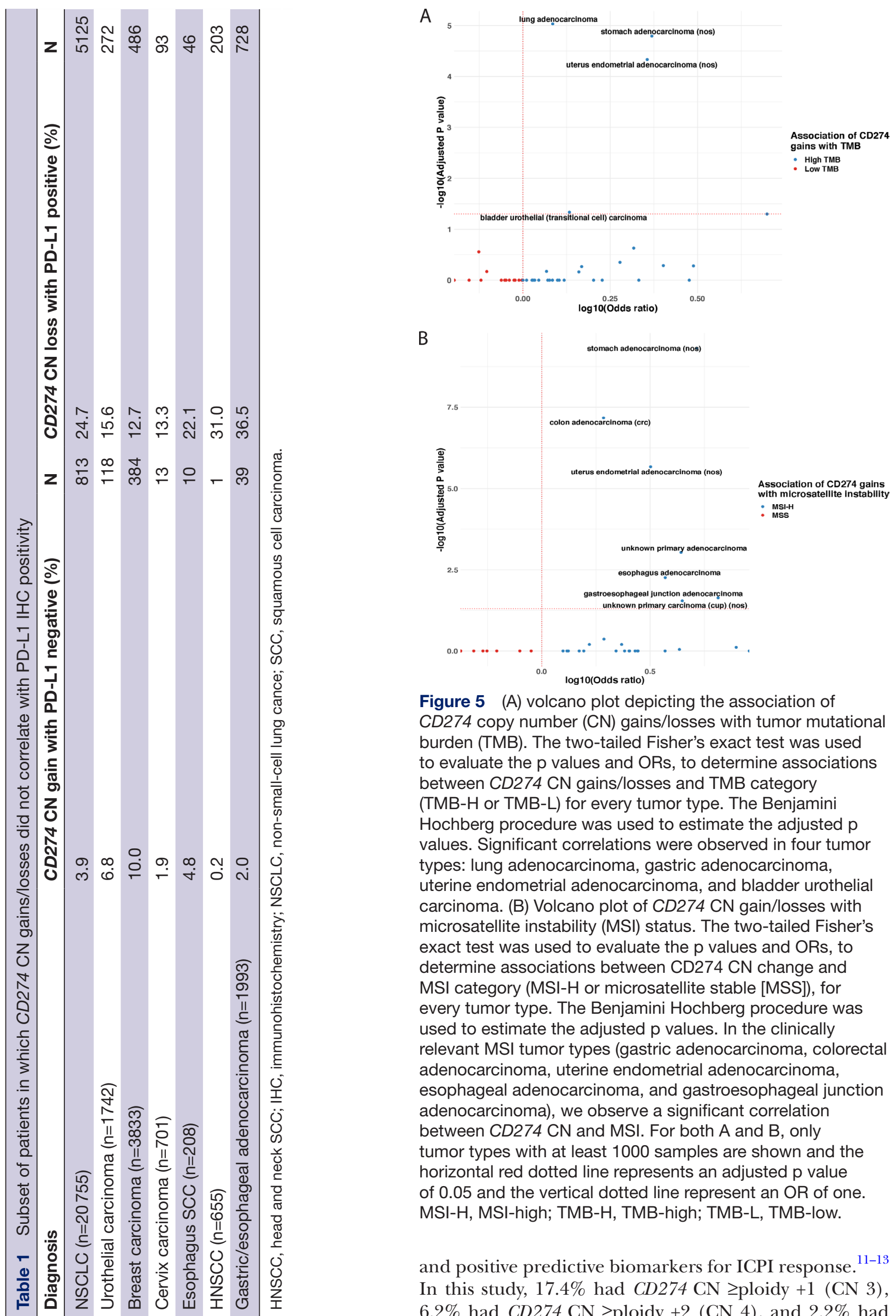

Figure 5 (A) volcano plot depicting the association of CD274 copy number (CN) gains/losses with tumor mutational burden (TMB). The two-tailed Fisher's exact test was used to evaluate the $p$ values and ORs, to determine associations between CD274 CN gains/losses and TMB category (TMB-H or TMB-L) for every tumor type. The Benjamini Hochberg procedure was used to estimate the adjusted $p$ values. Significant correlations were observed in four tumor types: lung adenocarcinoma, gastric adenocarcinoma, uterine endometrial adenocarcinoma, and bladder urothelial carcinoma. (B) Volcano plot of CD274 CN gain/losses with microsatellite instability (MSI) status. The two-tailed Fisher's exact test was used to evaluate the $p$ values and ORs, to determine associations between CD274 CN change and MSI category (MSI-H or microsatellite stable [MSS]), for every tumor type. The Benjamini Hochberg procedure was used to estimate the adjusted $p$ values. In the clinically relevant MSI tumor types (gastric adenocarcinoma, colorectal adenocarcinoma, uterine endometrial adenocarcinoma, esophageal adenocarcinoma, and gastroesophageal junction adenocarcinoma), we observe a significant correlation between $C D 274 \mathrm{CN}$ and MSI. For both $\mathrm{A}$ and $\mathrm{B}$, only tumor types with at least 1000 samples are shown and the horizontal red dotted line represents an adjusted $p$ value of 0.05 and the vertical dotted line represent an OR of one. MSI-H, MSI-high; TMB-H, TMB-high; TMB-L, TMB-low.

and positive predictive biomarkers for ICPI response. ${ }^{11-13}$ In this study, $17.4 \%$ had $\mathrm{CD} 274 \mathrm{CN} \geq$ ploidy +1 (CN 3), $6.2 \%$ had $C D 274 \mathrm{CN} \geq$ ploidy $+2(\mathrm{CN} 4)$, and $2.2 \%$ had 
CD274 CN $\geq$ ploidy $+3(\mathrm{CN} 5)$ across tumor types, which is 25-fold, 9-fold, and 3-fold higher, respectively, than when using CD274 $\mathrm{CN} \geq$ ploidy +4 (CN 6), where Goodman et $a l^{0}$ only found $0.7 \%$ of solid tumors in their cohort as amplified. Of note, in our current cohort, we found that $1.1 \%(2712 / 244584)$ had a $\mathrm{CN} \geq$ ploidy +4 (CN $6)$, which is higher than the $0.7 \%(843 / 118$ 187) that Goodman $e t a l^{10}$ described. The prevalence in our study likely more accurately describes the real-world prevalence since our data set has twice the number of samples when compared with the previous study. Future clinical trials with outcomes data are needed to assess the optimal CD274 CN cut-off for a patient to be considered positive and whether $\mathrm{CD} 274 \mathrm{CN}$ loss is a negative predictor for ICPI response in all tumor types or only certain tumor types. Given the varied levels of $\mathrm{CN}$ changes in the various tumor types presented in this study, we suspect that the $\mathrm{CN}$ cut offs that are correlated with ICPI response will vary based on tumor type. The prevalence and diversity of $C D 274 \mathrm{CN}$ changes in this study can serve as a basis for future clinical studies when further exploring $\mathrm{CD} 274 \mathrm{CN}$ changes.

Both gains and losses of $C D 274$ were correlated with PD-L1 IHC status. This stands in contrast to genes like ROS1, where CN changes and ROS1 protein expression detected via IHC are not highly correlated. ${ }^{27}$ Instead, CD274 CN gains are more similar to ERBB2 (HER2) $\mathrm{CN}$ gains and HER2 protein expression in that they are correlated with each other. ${ }^{27}$ Interestingly, HNSCC CD274 CN gains had the highest correlation with PD-L1 IHC positivity. Furthermore, the highest levels of $\mathrm{CN}$ gains were in tumor types with SCC morphology, suggesting that $C D 274$ $\mathrm{CN}$ gains could be a particularly useful biomarker for tumors with this morphology. In our exploratory analysis of HNSCC and cervical SCC, we saw a positive correlation of HPV infection with $C D 274 \mathrm{CN}$ gain in these two tumor types. This suggests that the HPV infection likely caused the higher prevalence of CD274 CN gains in HNSCC and cervical SCC, though the exact mechanism for this remains elusive and warrants further investigation.

Lastly, when we analyzed the correlation of TMB-H with CD274 CN gains, we found that in almost all tumor types, there was no significant correlation between $C D 274 \mathrm{CN}$ gains and TMB-H which is consistent with the findings by Yarchoan $e t a l^{28}$ that PD-L1 expression and TMB are independent biomarkers in most tumor types. On the other hand, we saw significant correlation between $C D 274 \mathrm{CN}$ gains and MSI-H in the tumor types where MSI is most clinically relevant, but not in most other tumor types. Importantly, subsets of patients were negative for PD-L1 IHC but had CD274 CN gain (also positive for PD-L1 IHC and had CD274 loss). Also, we saw high specificity and positive predictive value of $\mathrm{CD} 274 \mathrm{CN}$ positivity (with most CN cut-offs) with PD-L1 IHC positivity suggesting that $C D 274 \mathrm{CN}$ positivity is selecting patients who are likely to respond to ICPI. On the other hand, we observed relatively low sensitivity and negative predictive value of CD274 CN positivity (at almost all CN cut-offs), meaning that $\mathrm{CD} 274 \mathrm{CN}$ positivity is only selecting a subset of the PD-L1 IHC positive tumors. These results in whole suggest that $C D 274 \mathrm{CN}$ changes could be an independent positive or negative predictive biomarker for ICPI response.

\section{CONCLUSION}

CD274 CN changes and PD-L1 expression were highly correlated in multiple tumor types. CGP-based $C D 274 \mathrm{CN}$ losses/gains obtained during routine clinical care could identify subsets of patients that are discordant with other known ICPI biomarkers, supporting further development of $C D 274 \mathrm{CN}$ losses/gains as a ICPI biomarker. These prevalence data on $\mathrm{CD} 274 \mathrm{CN}$ changes across a large cohort of different solid tumors can be used to design future clinical studies to assess whether $C D 274 \mathrm{CN}$ changes could be a potential biomarker for ICPI.

Contributors Conception/design: RSPH, KM, JSR. Provision of study materia or patients: RSPH, KM, MM, DCP, DAM, MH, BD, GF, LAA, JSR. Collection and/ or assembly of data: RSPH, KM. Data analysis and interpretation: All authors. Manuscript writing: All authors. Final approval of manuscript: all authors.

Funding The authors have not declared a specific grant for this research from any funding agency in the public, commercial or not-for-profit sectors.

Competing interests All authors of the manuscript are employees of Foundation Medicine, which is a wholly owned subsidiary of Roche and receives stock from Roche.

Patient consent for publication Not required.

Ethics approval This study was approved by the Western Institutional Review Board Protocol No. 20152817.

Provenance and peer review Not commissioned; externally peer reviewed.

Data availability statement All data relevant to the study are included in the article or uploaded as online supplemental information. The data generated by the research that supports our article will be provided in the supplements. Due to the risk of patient reidentification, we are unable to share the raw alteration data. Academic researchers can gain access to the data in this study by filling out a study review committee form and by contacting the corresponding author. For further questions please reach out to Karen Schorr, Chief Compliance Officer, Foundation Medicine, Cambridge, Massachusetts, USA (kschorr@foundationmedicine.com).

Supplemental material This content has been supplied by the author(s). It has not been vetted by BMJ Publishing Group Limited (BMJ) and may not have been peer-reviewed. Any opinions or recommendations discussed are solely those of the author(s) and are not endorsed by BMJ. BMJ disclaims all liability and responsibility arising from any reliance placed on the content. Where the content includes any translated material, BMJ does not warrant the accuracy and reliability of the translations (including but not limited to local regulations, clinical guidelines, terminology, drug names and drug dosages), and is not responsible for any error and/or omissions arising from translation and adaptation or otherwise.

Open access This is an open access article distributed in accordance with the Creative Commons Attribution Non Commercial (CC BY-NC 4.0) license, which permits others to distribute, remix, adapt, build upon this work non-commercially, and license their derivative works on different terms, provided the original work is properly cited, appropriate credit is given, any changes made indicated, and the use is non-commercial. See http://creativecommons.org/licenses/by-nc/4.0/.

\section{ORCID iD}

Richard S.P. Huang http://orcid.org/0000-0001-8395-5168

\section{REFERENCES}

1 FDA. List of cleared or Approved companion diagnostic devices (in vitro and imaging tools), 2021. Available: https://www.fda. gov/medical-devices/vitro-diagnostics/list-cleared-or-approvedcompanion-diagnostic-devices-vitro-and-imaging-tools 
2 Gjoerup O, Brown CA, Ross JS, et al. Identification and utilization of biomarkers to predict response to immune checkpoint inhibitors. Aaps J 2020;22:132.

3 Huang RSP, Haberberger J, Severson E, et al. A pan-cancer analysis of PD-L1 immunohistochemistry and gene amplification, tumor mutation burden and microsatellite instability in 48,782 cases. Mod Pathol 2021;34:252-263.

4 Huang RSP, Li X, Haberberger J, et al. Biomarkers in breast cancer: an integrated analysis of comprehensive genomic profiling and PD-L1 immunohistochemistry biomarkers in 312 patients with breast cancer. Oncologist 2020;25:943-53.

5 Marabelle A, Fakih M, Lopez J, et al. Association of tumour mutational burden with outcomes in patients with advanced solid tumours treated with pembrolizumab: prospective biomarker analysis of the multicohort, open-label, phase 2 KEYNOTE-158 study. Lancet Oncol 2020;21:1353-65.

6 Sivapiragasam A, Ashok Kumar P, Sokol ES, et al. Predictive biomarkers for immune checkpoint inhibitors in metastatic breast cancer. Cancer Med 2021;10:53-61.

7 Ansell SM, Lesokhin AM, Borrello I, et al. Pd-1 blockade with nivolumab in relapsed or refractory Hodgkin's lymphoma. $N$ Engl $J$ Med 2015;372:311-9.

8 Armand P, Shipp MA, Ribrag V, et al. Programmed death-1 blockade with pembrolizumab in patients with classical Hodgkin lymphoma after Brentuximab Vedotin failure. J Clin Oncol 2016;34:3733-9.

9 Roemer MGM, Advani RH, Ligon AH, et al. Pd-L1 and PD-L2 genetic alterations define classical Hodgkin lymphoma and predict outcome. J Clin Oncol 2016;34:2690-7.

10 Goodman AM, Piccioni D, Kato S, et al. Prevalence of PDL1 amplification and preliminary response to immune checkpoint blockade in solid tumors. JAMA Oncol 2018:4:1237-44.

11 Bachelot T, Filleron T, Dalenc F, et al. 1280 PDL1/CD274 gain/ amplification as a predictive marker of checkpoint blockade inhibitor efficacy in metastatic breast cancer: Exploratory analysis of the SAFIR02-IMMUNO randomized phase II trial. Annals of Oncology 2020;31:S58-9.

12 Lamberti G, Spurr LF, Li Y, et al. Clinicopathological and genomic correlates of programmed cell death ligand 1 (PD-L1) expression in nonsquamous non-small-cell lung cancer. Ann Oncol 2020;31:807-14.

13 Inoue $\mathrm{Y}$, Yoshimura K, Nishimoto K, et al. Evaluation of programmed death ligand 1 (PD-L1) gene amplification and response to nivolumab monotherapy in non-small cell lung cancer. JAMA Netw Open 2020;3:e2011818.

14 Frampton GM, Fichtenholtz A, Otto GA, et al. Development and validation of a clinical cancer genomic profiling test based on massively parallel DNA sequencing. Nat Biotechnol 2013;31:1023-31.
15 Sun JX, He Y, Sanford E, et al. A computational approach to distinguish somatic vs. germline origin of genomic alterations from deep sequencing of cancer specimens without a matched normal. PLoS Comput Biol 2018;14:e1005965.

16 Chalmers ZR, Connelly CF, Fabrizio D, et al. Analysis of 100,000 human cancer genomes reveals the landscape of tumor mutational burden. Genome Med 2017:9:34.

17 FDA. FDA approves pembrolizumab for adults and children with TMB-H solid tumors, 2020. Available: https://www.fda.gov/drugs/ drug-approvals-and-databases/fda-approves-pembrolizumabadults-and-children-tmb-h-solid-tumors

18 Trabucco SE, Gowen K, Maund SL, et al. A novel next-generation sequencing approach to detecting microsatellite instability and PanTumor characterization of 1000 microsatellite Instability-High cases in 67,000 patient samples. J Mol Diagn 2019;21:1053-66.

19 FDA. FDA grants accelerated approval to pembrolizumab for first tissue/site agnostic indication, 2020. Available: https://www.fda. gov/drugs/resources-information-approved-drugs/fda-grantsaccelerated-approval-pembrolizumab-first-tissuesite-agnosticindication

20 VENTANA. VENTANA PD-L1 (SP142) assay package insert, 2019. Available: https://www.accessdata.fda.gov/cdrh_docs/pdf16/ p160002s009c.pdf

21 DAKO. PD-L1 IHC 22C3 pharmDx package insert, 2020. Available: https://www.accessdata.fda.gov/cdrh_docs/pdf15/P150013c.pdf

22 Systems VM. VENTANA PD-L1 (SP142) assay interpretation guide for triple-negative breast carcinoma (TNBC), 2019. Available: https:// diagnostics.roche.com/content/dam/diagnostics/us/en/resourcecenter/VENTANA-PD-L1-(SP142)-Assay-Interpretation-Guide.pdf

23 DAKO. PD-L1 IHC 22C3 pharmDx interpretation manual - NSCLC, 2019. Available: https://www.agilent.com/cs/library/usermanuals/ public/29158_pd-11-ihc-22C3-pharmdx-nsclc-interpretation-manual. pdf

24 DAKO. PD-L1 IHC 22C3 pharmDx interpretation manual - urothelia carcinoma, 2021. Available: https://www.agilent.com/cs/library/ usermanuals/public/29276_22C3_pharmdx_uc_interpretation_ manual us.pdf

25 Ihaka R, Gentleman R. R: a language for data analysis and graphics. Journal of Computational and Graphical Statistics 1996:5:299-314.

26 Gv R. Python reference manual, 2003. Available: https://docs.python org/2.2/ref/ref.htm

27 Huang RSP, Gottberg-Williams A, Vang P, et al. Correlating ROS1 protein expression with ROS1 fusions, amplifications, and mutations. JTO Clin Res Rep 2021;2:100100.

28 Yarchoan M, Albacker LA, Hopkins AC, et al. PD-L1 expression and tumor mutational burden are independent biomarkers in most cancers. JCl Insight 2019;4. doi:10.1172/jci.insight.126908. [Epub ahead of print: 2103 2019]. 